\title{
ESTUDIO DE LA MADURACIÓN TERMAL DE KERÓGENO EN LOS CARBONES DE LA FORMACIÓN GUADUAS, CORDILLERA ORIENTAL (COLOMBIA), MEDIANTE ANÁLISIS DE REFLECTANCIA DE VITRINITA, PIROLISIS ROCK EVAL, RESONANCIA MAGNÉTICA NUCLEAR Y ANÁLISIS PROXIMAL
}

\author{
Tania S. Palmera-Henao ${ }^{1}$; Mario García-González ${ }^{1}$
}

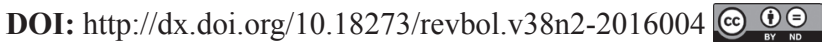

Forma de citar: Palmera-Henao, T., y García-González, M. 2016. Estudio de la maduración termal de kerógeno en los carbones de la Formación Guaduas, Cordillera Oriental (Colombia), mediante análisis de reflectancia de vitrinita, pirolisis Rock Eval, resonancia magnética nuclear y análisis proximal. Boletín de Geología, 38(2): 63-73.

\section{RESUMEN}

El presente trabajo reporta los resultados del estudio de los procesos de maduración termal de los kerógenos tipo II y III presentes en los mantos de carbón de la Formación Guaduas de edad Maestrichtiano a Paleoceno, en la Cordillera Oriental de Colombia. Los resultados de los análisis de maduración termal de la sección del Triunfo en el Sinclinal de Checua-Lenguazaque permitieron establecer: Reflectancia de vitrinita (\%Ro) entre 0,63 y $1,04 \%$; Tmax entre 439 y $459^{\circ} \mathrm{C}$; Materia Volátil (MV) $31 \%$ y $40 \%$; y factor de aromaticidad de RMN que varía entre 0,69 y $0,83 \%$. La metodología utilizada realizó una calibración con respecto a Ro de los diferentes análisis de maduración termal del kerógeno, obteniéndose las respectivas ecuaciones que permiten calcular las equivalencias entre los diferentes parámetros. Este método garantiza la correcta medición de la maduración termal de rocas fuente de hidrocarburos y es aplicable a otras formaciones y cuencas. Las correlaciones entre los parámetros empleados permiten calcular la maduración de los carbones del Cretáceo Superior y Paleoceno en donde se han publicado los análisis proximales de carbones a diferencia de los datos de Ro.

Palabras clave: kerógeno, maduración termal, Resonancia Magnética Nuclear (RMN), Reflectancia de Vitrinita (Ro), Tmax, Materia Volátil.

\section{THERMAL MATURATION STUDY OF KEROGEN IN THE COAL SEAMS OF THE GUADUAS FORMATION, EASTERN CORDILLERA (COLOMBIA), USING VITRINITE REFLECTANCE, ROCK EVAL PIROLISIS, NUCLEAR MAGNETIC RESONANCE AND PROXIMAL ANALYSIS}

\begin{abstract}
Geochemical studies of thermal maturation on kerogen types II and III are presented. This research was carried out on coal seams of the Guaduas Formation (Maastrichtian to Paleocene) outcropping in the Checua-Lenguazaque syncline, Eastern Cordillera of Colombia. The thermal maturation analyses indicate the following maturation ranges: Vitrinite Reflectance between 0.63 and $1.04 \%$; Tmax between 439 and $459^{\circ} \mathrm{C}$; and the Nuclear Magnetic Resonance (NMR) between 0.69 and $0.83 \%$; and Volatile matter VM between 31 and $40 \%$. In this study the different maturation parameters were calibrated in reference to Ro obtaining correlation equations that allows to calculate the equivalence between these parameters. This method measures the correct thermal maturation of hydrocarbon source rocks and can be applied to other formations and basins. The results obtained in this study allow calculating the thermal maturation of the Upper Cretaceous- Paleogene Coal-bearing formations of Colombia where proximate analyses but no Ro analyses have been published.
\end{abstract}

Keywords: kerogen, thermal maturation, Nuclear Magnetic Resonance (NMR), Vitrinite Reflectance (Ro), Tmax, Volatile Matter.

${ }^{1}$ Universidad Industrial de Santander. Escuela de Geología. Bucaramanga, Santander, Colombia. Grupo de Investigación en Geología de Hidrocarburos y Carbones (G.I.G.H.C). taniahenao@hotmail.com, mgarciag@uis.edu.co, gighc@uis.edu.co. 


\section{INTRODUCCIÓN}

La Cordillera Oriental presenta un potencial de yacimientos no convencionales, cuyos recursos pueden incrementar las reservas de hidrocarburos de Colombia. Por esta razón es importante desarrollar técnicas de evaluación que permitan determinar el potencial de generación y de acumulación de hidrocarburos en yacimientos no convencionales. El presente trabajo de investigación evalúa el grado de maduración termal del kerógeno en los carbones de la Formación Guaduas, específicamente en la sección denominada como el Triunfo, localizada en el departamento de Cundinamarca, Cordillera Oriental, Colombia (FIGURA 1). La metodología empleada en este trabajo puede ser aplicada a yacimientos de tipo gas asociado al carbón y shale gas, en los cuales la relación entre el grado de madurez termal y el potencial de generación y acumulación de hidrocarburos se encuentra directamente proporcional, permitiendo de esta manera evaluar el potencial de reservas de estos yacimientos no convencionales.

Los trabajos de exploración de yacimientos no convencionales requieren establecer el grado de maduración termal de las rocas fuente, las cuales actúan a su vez como rocas reservorio. De esta manera, se identifica el potencial de generación y acumulación de petróleo, gas termogénico y gas biogénico. Los análisis de maduración termal del kerógeno en diferentes formaciones geológicas presentan alta variación dependiendo del tipo de materia orgánica y de la litología de la roca huésped del kerógeno, tal como se evidencia en esta investigación y en estudios previos realizados por Behar et al. (1997); Vanderbroucke and Largeau (2007). La reflectancia de vitrinita (Ro) es quizá uno de los parámetros más confiables para la determinación del grado de madurez termal; sin embargo, el Ro no siempre se puede medir cuando las partículas de vitrinita o vitrinoides presentan un reducido tamaño que impide aislar la luz reflejada por el maceral para su respectiva medición cuantitativa. De igual manera, los valores de Tmax se pueden afectados por la matriz mineral de la roca huésped y por la naturaleza del kerógeno presente.

La resonancia magnética nuclear (RMN) es un parámetro adicional que permite medir el grado de madurez termal a partir de la cuantificación de la aromatización (fa) del kerógeno presente en las rocas fuente de hidrocarburo. En el presente artículo se reportan nuevos datos de mediciones de maduración termal (Ro, Tmax, fa y análisis proximal) en la Formación Guaduas, los cuales permiten establecer equivalencias entre los diferentes parámetros de madurez termal.

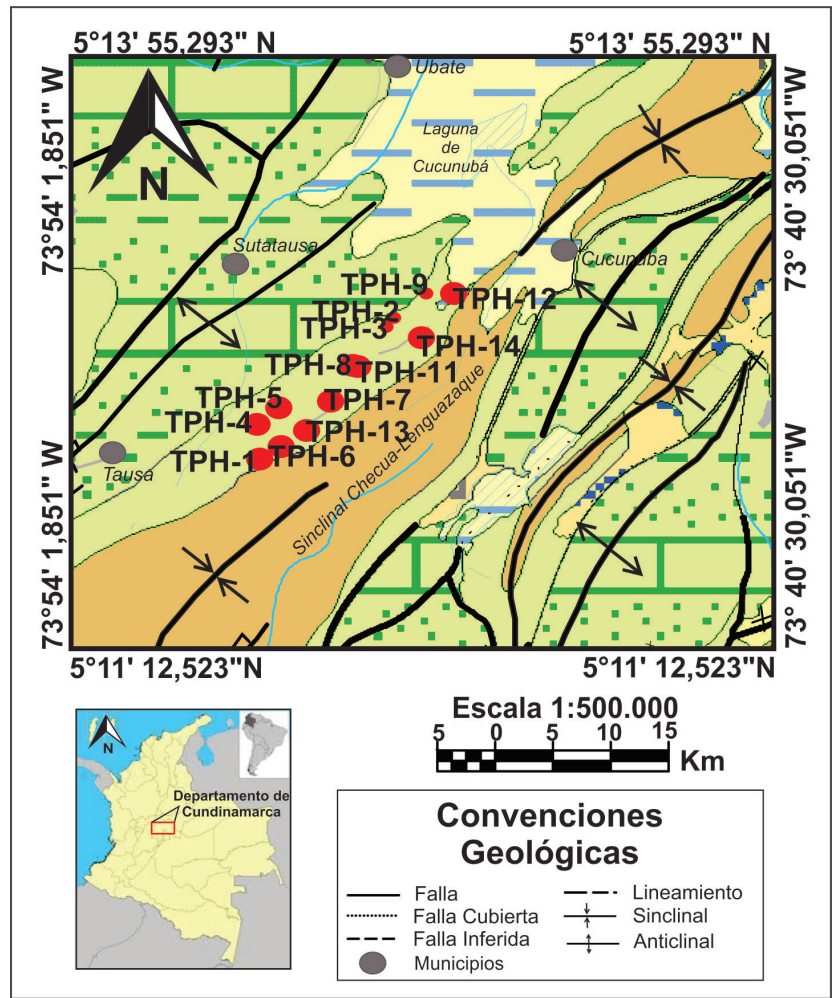

FIGURA 1. Mapa de localización de la sección del Triunfo, Sinclinal Checua-Lenguazaque, departamento de Cundinamarca, Colombia. Modificado de Atlas Geológico de Colombia (Gómez et al., 2007). 


\section{METODOLOGÍA}

Con el objeto de llevar a cabo la caracterización geoquímica y analizar los procesos de maduración termal del kerógeno presente en la formación seleccionada, se llevó a cabo el respectivo muestreo en mantos de carbón de la Formación Guaduas (mantos 1 al 19) localizados en el Sinclinal Checua-Lenguazaque, sección del Triunfo (FIGURA 2), cuyos frentes de explotación se encontraran en producción, con base en la columna estratigráfica levantada por Rincón y Rocha (2007).

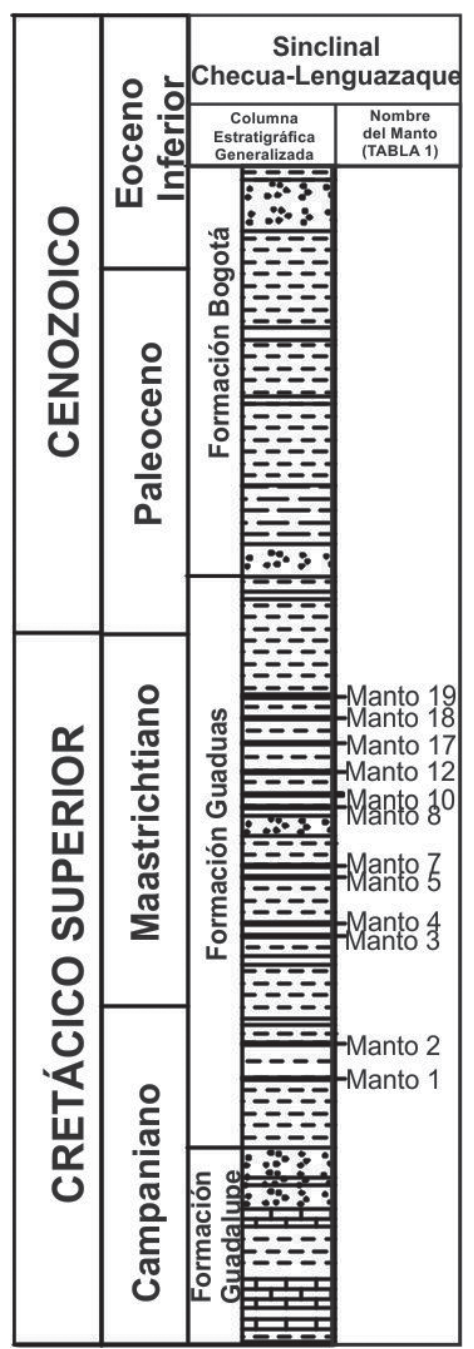

FIGURA 2. Columna estratigráfica generalizada, Sección del Triunfo, Sinclinal Checua-Lenguazaque. Modificado de Rincón y Rocha (2007).

El análisis de las muestras recolectadas en campo se realizó en los laboratorios de la Universidad Industrial de Santander, Colombia, por medio de las siguientes técnicas: Carbono orgánico total (\%TOC), pirolisis Rock Eval, reflectancia de vitrinita (\%Ro), resonancia magnética nuclear (RMN) y análisis proximales.
La determinación de carbono orgánico total (\%TOC) se llevó a cabo en el equipo analizador de carbono y azufre LECO SC-144DR, usando el método de carbono orgánico total (\%TOC) mediante un horno de combustión de alta temperatura $\left(1.350^{\circ} \mathrm{C}\right)$ con detección infrarroja (IR) no dispersiva. El resultado es expresado a modo de porcentaje (\%). En esta técnica la muestra es sometida a una temperatura máxima de $1.350{ }^{\circ} \mathrm{C}$, en una atmosfera oxidante durante aproximadamente dos minutos, tiempo suficiente para que los vapores de la combustión de la muestra ( $\mathrm{CO}_{2}$ producido durante la combustión) pasen a través de la celda de espectro infrarrojo no dispersivo; determinando así la cantidad de TOC presente en cada una de las muestras (incluye tanto el kerógeno como el bitumen). El peso aproximado de las muestras para este análisis fue $0,01 \mathrm{~g}$.

Sin embargo, se debe tener presente que el porcentaje de carbono orgánico total obtenido en cada una de las muestras no es por sí solo un indicador del potencial o la capacidad de una roca para generar hidrocarburos. Se recomienda correlacionar los resultados obtenidos con análisis de pirolisis Rock Eval y reflectancia de vitrinita (Ro).

Los análisis de resonancia magnética nuclear (RMN) en muestras sólidas, se realizaron en el equipo ultrashield 400, modelo Bruker de RMN, bajo los siguientes parámetros: secuencia one pulse, frecuencia de 14 MHz para 13C y un ángulo de rotación (MAS) de 544 ', (Miknis et al., 1981; Stéphane et al., 1981; Werner et al., 2005). Los espectros resultantes del análisis fueron tratados mediante dos programas: el programa Mestre-c versión 4.9.9.6 y el programa Dmfit de la universidad D'Orleans en Francia (versión libre). Por medio del programa Mestre-c versión 4.9.9.6, se hizo el alisamiento de los espectros y la posterior integración de las áreas aromáticas y alifáticas para calcular el factor de aromaticidad (fa), ver ecuación (1), teniendo en cuenta los límites dados por Snape (1979) en Van Krevelen (1993).

$\mathrm{fa}=\frac{\text { carbono aromatico }}{\text { carbono aromatico }+ \text { carbono alifatico }}$

Ecuación (1)

Por otra parte, el programa Dmfit de la universidad D'Orleans en Francia (versión libre), fue usado para realizar las deconvoluciones de los espectros de cada una de las muestras analizadas y determinar así los grupos funcionales que acompañan a los carbonos aromáticos y alifáticos de cada muestra, respectivamente.

La medición y análisis de reflectancia de vitrinita (\%Ro) se llevó a cabo mediante el microscopio Axiotron 
MPM400 Carl Zeiss. La calibración del instrumento se realizó empleando estándares de reflectancia de $0,54 \%$ y $1,71 \%$ con luz reflejada, cuya longitud de onda es de 546 nm. A si mismo, los datos obtenidos fueron procesados mediante el software Photan (software del fotómetro) y expresados como \%Ro. Luego de la calibración del equipo, se tomaron 30 lecturas de \%Ro por cada sección pulida (cada muestra); y así se identificaron claramente los macérales de vitrinita sobre la superficie pulida, teniendo el cuidado de no tomar lecturas en macérales diferentes a la vitrinita o vitrinitas de bajo tamaño que pudieran causar interferencia en su aislamiento para la respectiva medición, pues de lo contrario se alterarían los resultados. La desviación estándar de las 30 lecturas de \%Ro por cada sección pulida (cada muestra) fue inferior a 0,003 .

En esta investigación, \%Ro proporcionó datos contundentes sobre el grado de madurez termal de las muestras analizadas (mantos de carbón), debido a que la vitrinita (maceral) va aumentando su reflectividad, a causa de reacciones de aromatización irreversibles y complejas, en la medida que aumenta la madurez termal de la roca. Dichas reacciones irreversibles han sido reportadas en investigaciones realizadas por Van Krevelen (1993), Bustin and Guio (1999), y Vanderbroucke and Largeau (2007).

Por otra parte, el procedimiento analítico realizado mediante la técnica de pirolisis Rock Eval se llevó a cabo en el equipo analizador anhídrido de muestras de roca y suelo Rock Eval, versión Turbo 6, programado para ciclo completo de análisis, es decir, pirolisis y oxidación. El peso aproximado de las muestras para este análisis fue de 10 a $20 \mathrm{mg}$.

Los productos que se obtienen durante el proceso analítico de pirolisis Rock Eval son medidos en tiempo real por celdas infrarrojas (IR), obteniendo así resultados de los siguientes parámetros: Pico S1, medida de los hidrocarburos libres expresado en miligramos de hidrocarburos ( $\mathrm{HC}$ ) / gramos roca ( $\mathrm{mg} \mathrm{HC} / \mathrm{g}$ roca); pico $\mathrm{S} 2$, correspondiente a la medida de los hidrocarburos producidos durante el calentamiento por craqueo del kerógeno, expresado en $\mathrm{mg} \mathrm{HC/g}$ roca; Tmax o temperatura máxima alcanzada durante el craqueo del kerógeno en el pico $\mathrm{S} 2$ y el pico $\mathrm{S} 3$, correspondiente a la medida de $\mathrm{CO}$ y $\mathrm{CO}_{2}$ producido durante la pirolisis,

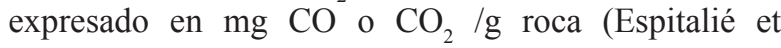
Marquis, 1992). Así mismo, durante el proceso analítico se obtienen medidas del índice de producción (IP), índice de hidrógeno (IH) e índice de oxigeno (IO) en tiempo real (Espitalié et Marquis, 1992).
El análisis proximal de carbones funciona como un parámetro de madurez termal. Sin embargo, se recomienda correlacionar este parámetro con datos de Ro, RMN y pirolisis Rock Eval. Para los análisis proximales, las muestras deben ser previamente trituradas, tamizadas y maceras en un mortero de Agatha. Estos análisis se desarrollaron bajo los lineamientos de las normas ASTM D3173, ASTM D4239b, ASTM D3174 y ASTM D3173. Se obtuvieron datos de porcentajes (\%) en peso de Humedad, Materia Volátil, Cenizas y Carbono Fijo de cada una de las muestras de carbón analizadas.

\section{RESULTADOS OBTENIDOS}

La TABLA 1 muestra detalladamente los resultados obtenidos de los análisis realizados a las muestras de mantos de carbón de la Formación Guaduas, localizados en el Sinclinal de Checua-Lenguazaque (sección del Triunfo), Departamento de Cundinamarca, Colombia (FIGURA 1). Las técnicas usadas para tal fin fueron las siguientes: reflectancia de vitrinita, pirolisis Rock Eval, carbono orgánico total, materia volátil y resonancia magnética nuclear. En la discusión de resultados se explica en profundidad la tendencia de los mismos, a fin de analizar el grado de maduración termal del kerógeno en los carbones de esta formación.

\section{Reflectancia de vitrinita}

Los resultados de la reflectancia de vitrinita (Ro) obtenidos de los 14 mantos de carbón muestreados de la Formación Guaduas, muestran una tendencia incremental del Ro hacia la base de la Formación (TABLA 1).

Con base en las mediciones de Ro, se logro identificar la etapa de maduración en la cual se encuentran los mantos de carbón de esta sección. Los mantos inferiores muestran valores incrementales con la profundidad variando entre 0,9 y $1,35 \%$ Ro; mientras que los mantos superiores muestran valores que incrementan con la profundidad y cuyos valores varían entre 0,65 y $0,9 \%$ (FIGURA 2).

En las FIGURAS 3, 4 y 5 se evidencian macerales de vitrinita representativos de las muestras de mantos inferiores (manto 1), intermedios (manto 8) y superiores (manto 18), ver columna estratigráfica en la FIGURA 2. De igual forma, en el diagrama de barras o histograma que acompaña a las FIGURAS 3, 4 y 5, se muestran los diferentes intervalos de medición que se obtuvieron a partir de las 30 lecturas de \%Ro realizadas para cada muestra respectivamente. 
TABLA 1. Resultados de los análisis geoquímicos realizados a muestras de mantos de carbón de la Formación Guaduas, sección Triunfo.

\begin{tabular}{|c|c|c|c|c|c|c|c|c|c|c|c|c|}
\hline \multicolumn{13}{|c|}{ RESULTADOS DE ANÁLISIS GEOQUÍMICOS PARA LA SECCIÓN DEL TIRUNFO } \\
\hline \multirow{2}{*}{$\begin{array}{l}\text { NOMBRE } \\
\text { DE LA } \\
\text { MUESTRA }\end{array}$} & \multirow{2}{*}{$\begin{array}{c}\text { *NOMBRE } \\
\text { DEL } \\
\text { MANTO }\end{array}$} & \multirow{2}{*}{$\begin{array}{c}\% \\
\text { TOC }\end{array}$} & \multicolumn{4}{|c|}{$\begin{array}{ccc} & \text { REFLECTANCIA } & \\
\text { RMN } & \text { DE VITRINITA } & \text { ANÁLISIS } \\
& \text { PROXIMAL } \\
& (\% \text { Ro }) & \end{array}$} & \multicolumn{6}{|c|}{ PIROLISIS ROCK EVAL } \\
\hline & & & $\%$ fa & Promedio & $\begin{array}{c}\text { Materia } \\
\text { Volátil } \\
\text { (\%en peso) } \\
\end{array}$ & $\begin{array}{c}\mathrm{S1} \\
(\mathrm{mg} / \mathrm{g})\end{array}$ & $\begin{array}{c}\mathrm{S} 2 \\
(\mathrm{mg} / \mathrm{g})\end{array}$ & $\begin{array}{c}\mathrm{S3} \\
(\mathrm{mg} / \mathrm{g})\end{array}$ & $\begin{array}{c}\text { Tmax } \\
\left({ }^{\circ} \mathrm{C}\right)\end{array}$ & PI & HI & OI \\
\hline TPH-1 & Manto 1 & 84.620 & 0,769 & 0,9545 & 33,4719 & 3,56 & 217,62 & 0,45 & 450 & 0,02 & 257 & 1 \\
\hline TPH-3 & Manto 2 & 84.342 & 0,781 & 0,9223 & 35,6222 & 8,56 & 201,30 & 0,69 & 448 & 0,04 & 239 & 1 \\
\hline TPH-4 & Manto 3 & 87.788 & 0,833 & 1,0448 & 31,3443 & 4,74 & 205,51 & 0,51 & 452 & 0,02 & 234 & 1 \\
\hline TPH-5 & Manto 4 & 87.406 & 0,793 & 0,9475 & 31,0926 & 5,95 & 172,76 & 1,93 & 450 & 0,03 & 198 & 2 \\
\hline TPH-6 & Manto 5 & 77.735 & 0,787 & 0,8829 & 35,4103 & 5,95 & 202,57 & 1,54 & 446 & 0,03 & 261 & 2 \\
\hline TPH-7 & Manto 7 & 82.525 & N.A & 0,8942 & 36,6481 & 8,24 & 222,28 & 1,46 & 443 & 0,04 & 269 & 2 \\
\hline TPH-8 & Manto 11 & 70.696 & 0,699 & 0,8581 & 35,6293 & 5,45 & 193,27 & 2,44 & 449 & 0,03 & 273 & 3 \\
\hline TPH-9 & Manto 8 & 77.351 & 0,763 & 0,9445 & 33,3729 & 6,75 & 202,68 & 2,20 & 452 & 0,03 & 262 & 3 \\
\hline ТPH-10 & Manto 10 & 83.142 & 0,763 & 0,9511 & 34,0132 & 5,37 & 211,43 & 1,65 & 443 & 0,02 & 254 & 2 \\
\hline ТРН-11 & Manto 12 & 83.061 & N.A & 0,8397 & 38,5708 & 5,17 & 258,66 & 0,98 & 444 & 0,02 & 311 & 1 \\
\hline ТРН-12 & Manto 17 & 78.053 & N.A & 0,7457 & 39,1905 & 11,75 & 236,42 & 0,87 & 437 & 0,05 & 303 & 1 \\
\hline ТРН-13 & Manto 18 & 78.333 & N.A & 0,7456 & 39,4025 & 6,27 & 271,17 & 2,62 & 436 & 0,02 & 346 & 3 \\
\hline TPH-14 & Manto 19 & 76.797 & N.A & 0,6326 & 40,5221 & 8,74 & 252,77 & 2,38 & 435 & 0,03 & 329 & 3 \\
\hline
\end{tabular}

N.A= Análisis aplicados a la muestra. *El manto 1 presenta mayor profundidad (950m) y el manto 19 es el más superficial (100 m).
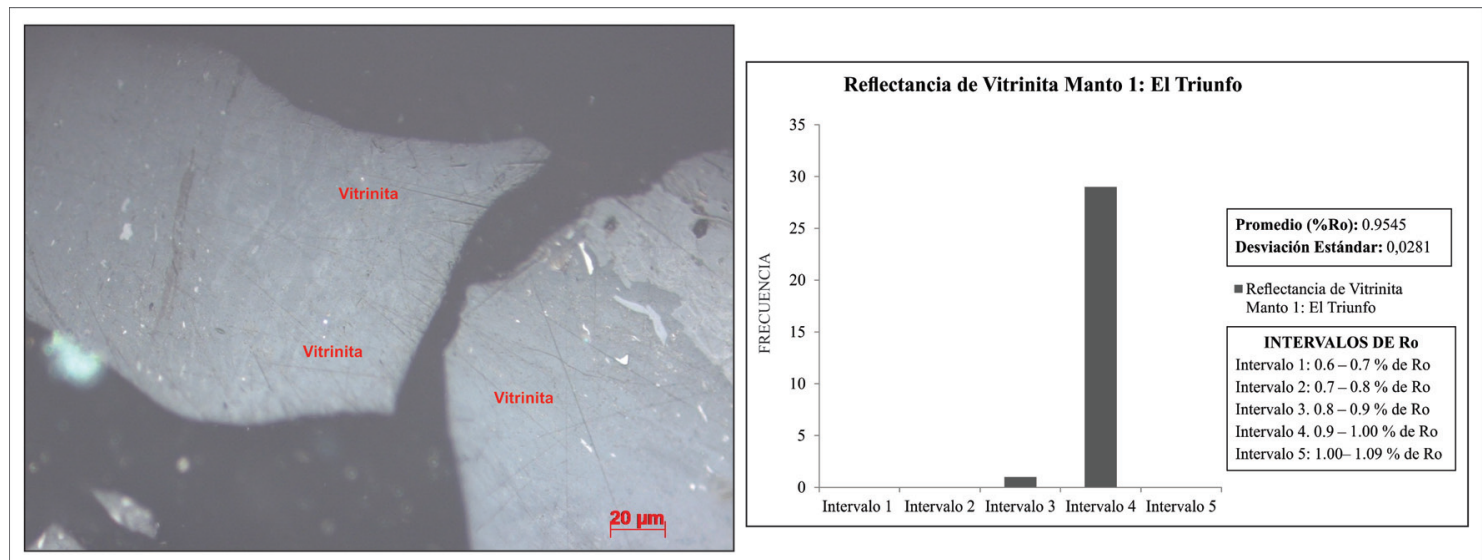

FIGURA 3. Macerales de vitrinita representativos del manto de carbón 1 (manto inferior) y diagrama de frecuencia de medición de \%Ro, sección del Triunfo.
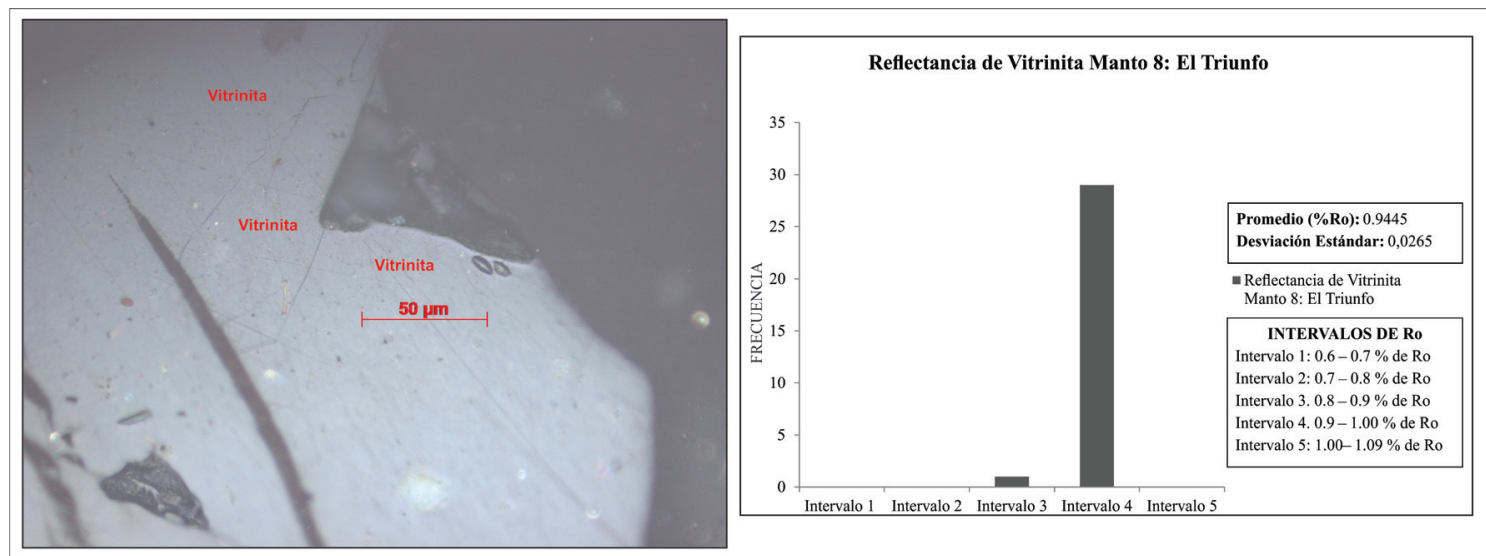

FIGURA 4. Macerales de vitrinita representativos del manto de carbón 8 (manto intermedio) y diagrama de frecuencia de medición de \%Ro, sección del Triunfo. 

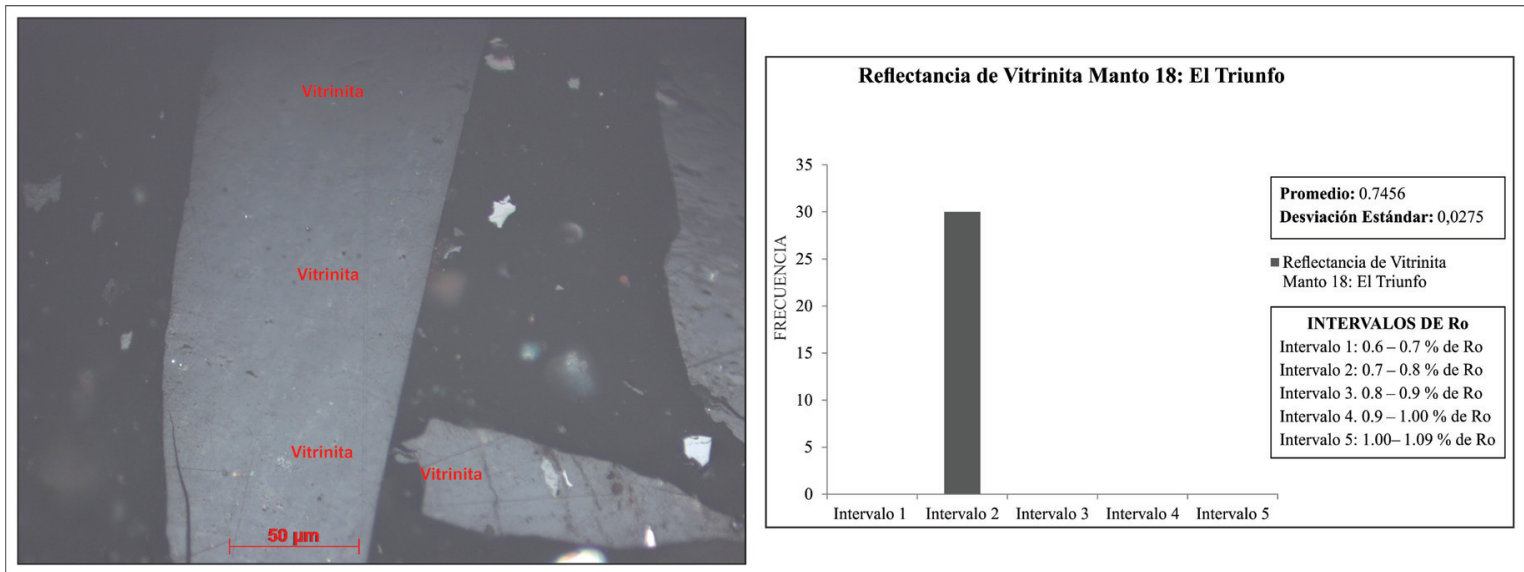

FIGURA 5. Macerales de vitrinita representativos del manto de carbón 18 (manto superior) y diagrama de frecuencia de medición de \%Ro, sección del Triunfo.

\section{Pirolisis Rock Eval}

Los análisis de pirolisis Rock Eval se obtuvieron con el equipo Turbo 6. El parámetro Tmax fue empleado como indicador de madurez termal. Los resultados de Tmax muestran una tendencia incremental de la temperatura de Tmax hacia la base de la Formación Guaduas (TABLA 1).

De acuerdo con lo anterior, los mantos inferiores muestran valores de Tmax que varían entre 450 y 470 ${ }^{\circ} \mathrm{C}$; mientras que los mantos superiores muestran valores de Tmax que varían entre 435 y $445^{\circ} \mathrm{C}$.

Los resultados obtenidos de los índices de hidrogeno (IH) y oxigeno (IO), permitieron identificar que los mantos de carbón de la Formación Guaduas, están constituidos por kerógeno tipo III y kerógeno tipo II; de acuerdo con los resultados obtenidos mediante el programa Rockint del equipo de pirolisis Rock Eval turbo 6. De igual forma, la correlación del IH versus la cantidad de materia volátil ( $\%$ en peso) presente en los diferentes mantos de carbón, evidencia que los mantos superiores presentan una alta cantidad de hidrogeno disponible en el kerógeno, mientras que los mantos inferiores tienden a disminuir la cantidad de hidrogeno a medida que aumenta el grado de madurez termal.

El aumento de la madurez termal produce una mayor aromatización y deshidrogenación del kerógeno, acompañado por pérdida de materia volátil; generando un incremento paulatino del grupo de carbonos aromáticos y una disminución del grupo carbonos alifáticos a medida que se aumenta la profundidad de enterramiento de los mantos de carbón ( FIGURA 6). La tendencia que se observa en la FIGURA 6 es correlacionable con los datos obtenidos de RMN y Ro.

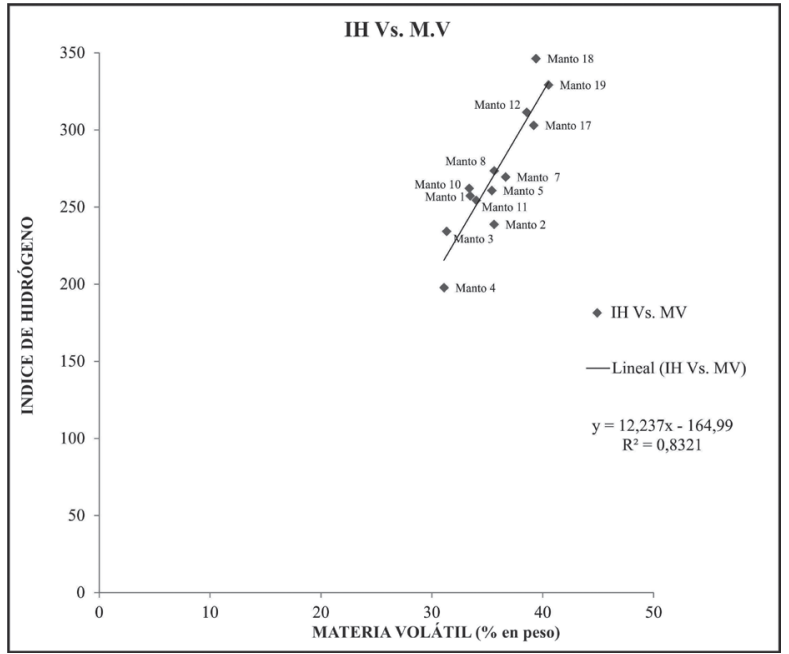

FIGURA 6. Índice de hidrógeno (IH) vs Materia Volátil (M.V).

\section{Carbono Orgánico Total (TOC) y materia volátil}

Los resultados obtenidos en los análisis de TOC, presentan una tendencia a aumentar hacia la base de la Formación Guaduas, explicada por la generación y expulsión de hidrocarburos alifáticos con la consecuente concentración de hidrocarburos aromáticos que conlleva a un aumento del contenido de carbono en el kerógeno. La investigación de Valenzuela y García (2002), reporta iguales resultados al someter muestras de carbón a procesos de hidropirólisis.

Los análisis proximales de carbones, muestran que el contenido de materia volátil tiende a disminuir hacia la base de la Formación Guaduas, indicando un aumento en el grado de maduración termal, el cual es explicado por la generación y expulsión de hidrocarburos (TABLA 1). 


\section{Resonancia Magnética Nuclear (RMN)}

El factor de aromaticidad (fa) obtenido a partir de análisis de RMN en muestras de carbones, es otro parámetro de madurez termal. Los resultados muestran una tendencia a aumentar hacia la base de la Formación Guaduas, la cual se explica con el incremento del grado de madurez termal. Los espectros de los mantos de carbón obtenidos en RMN muestran de forma generalizada un incremento en tamaño y nitidez en el pico del carbono aromático (FIGURA 7, pico de la izquierda) a medida que aumenta el grado de maduración termal. El carbono aromático se encuentra ubicado dentro del intervalo de desplazamiento químico de 100 a 180 ppm (Van Krevelen, 1993). Paralelamente, se observa un decrecimiento no muy marcado con menor nitidez en el pico del grupo alifático a medida que aumenta el grado de maduración termal (FIGURA 7, pico de la derecha); el carbono alifático se encuentra ubicado dentro del intervalo de desplazamiento químico de 11 a 60 ppm (Van Krevelen, 1993).

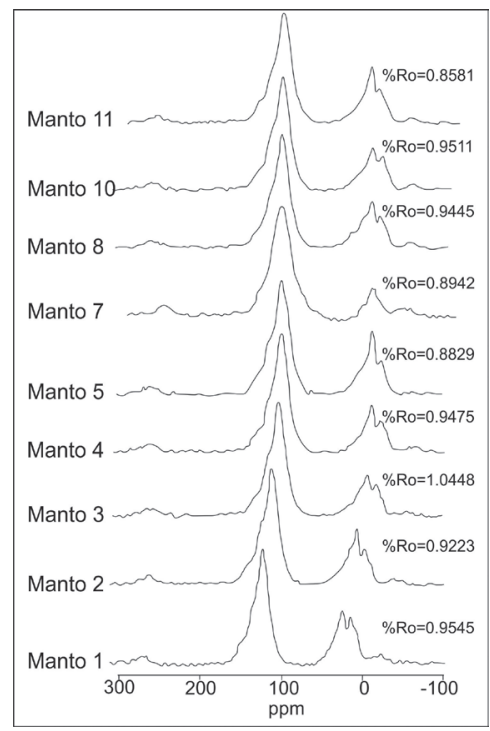

FIGURA 7. Espectros de RMN de los mantos de carbón analizados para la sección del Triunfo.

El análisis de deconvolución de los espectros de RMN mediante el programa Dmfit, muestra que el pico del enlace de carbono alifático disminuye con la profundidad de los mantos de carbón. De igual manera, se observa que el pico del enlace carbono aromático aumenta con la profundidad. Estas dos tendencias proporcionalmente inversas se explican por el incremento de la maduración termal de los mantos de carbón que conlleva a la generación y expulsión de hidrocarburos alifáticos y a la aromatización del kerógeno en el carbón. Se han reportado resultados semejantes para muestras de carbones y shales en la Formación Almond, Cuenca
Greater Green River en Wyoming por García-González et al. (1993).

De acuerdo con la deconvolución realizada en los espectros de RMN, mediante el programa Dmfit, donde cada pico enumerado en la FIGURA 8 corresponde a un grupo funcional especifico que acompaña los enlaces de carbonos aromáticos y alifáticos (TABLA 2); los mantos de carbón intermedios (mantos del 12 al 5), muestran mayor tamaño y nitidez en el pico del carbono aromático, junto con una disminución paulatina de intensidad del pico del grupo alifático. De igual forma, se observa la disminución de los grupos funcionales que acompañan al pico del carbono alifático, poniendo en evidencia la disminución de los picos número 2, 3, 4 y 5 , con base en la FIGURA 8 y la TABLA 2. El aumento de la condensación del kerógeno con la profundidad se muestra con la intensidad de los picos 9, 10 y 11 correspondiente a los enlaces aromáticos con sus respectivos grupos funcionales; de acuerdo con la FIGURA 8 y la TABLA 2.

En la TABLA 2 se identifica cada uno de los grupos funcionales que acompañan a los enlaces de carbonos alifáticos y aromáticos, respectivamente.

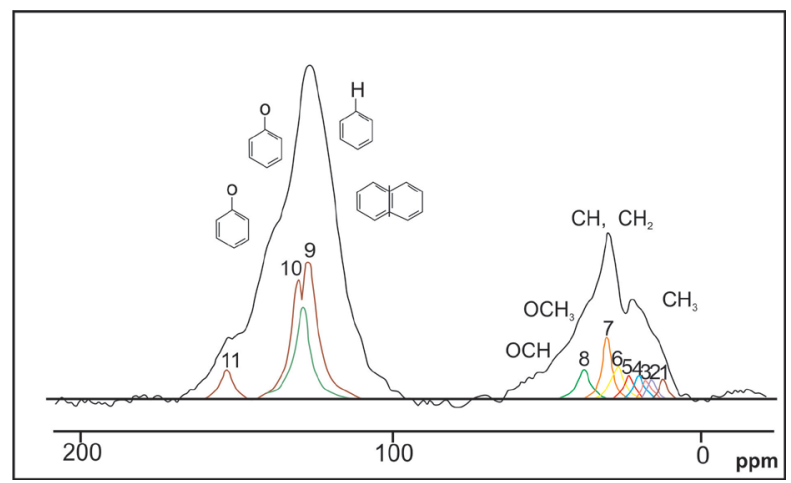

FIGURA 8. Deconvolución del espectro de RMN para la muestra del manto de carbón 8 (manto intermedio), mediante el programa Dmfit de la Universidad D'orleans de Francia (versión gratuita).

En los carbones de la base de la Formación Guaduas (mantos 1 al 4), los grupos funcionales y radicales que acompañan los enlaces de los carbonos alifaticos tienden a disminuir notablemente con el aumento del grado de maduración termal. Se observa la desaparición del grupo funcinal $\mathrm{CH} 2$ junto a un grupo alquilo, en el intervalo de 24-27,5 ppm, el CH3 en hidroaromáticos y anillos naftalénicos, en el intervalo de 18-24 ppm (correspondiendo al pico número 5 en la FIGURA 9); además de la disminución de los grupos funcionales localizados en los picos numero: 1, 2, 4 y 8, de acuerdo con la FIGURA 9 y la TABLA 2. 
TABLA 2. Grupos funcionales que acompañan los enlaces de carbonos alifáticos y aromáticos en la figura 3. Con sus respectivos intervalos de desplazamiento.

\begin{tabular}{|c|c|}
\hline $\begin{array}{c}\text { Pico enumerado } \\
\text { en la figura } 3\end{array}$ & Grupo funcional químico identificado \\
\hline Pico número 1 & $\begin{array}{l}\text { CH3 ó } \alpha-\text { CH3 alejados de anillos aromáticos por dos anillos o grupos } \\
11-15 \text { ppm. }\end{array}$ \\
\hline Pico numero 2 & $\beta-\mathrm{CH} 3$ en grupos etilo, en el intervalo de $15-18 \mathrm{ppm}$. \\
\hline Pico numero 3 & $\alpha-\mathrm{CH} 3$ protegido por un anillo o grupo adyacente, en el intervalo de $18-20,5 \mathrm{ppm}$. \\
\hline Pico numero 4 & $\alpha-\mathrm{CH} 3$ no protegido por ningún anillo o grupo, en el intervalo de $20,5-22,5 \mathrm{ppm}$. \\
\hline Pico numero 5 & $\begin{array}{l}\mathrm{CH} 2 \text { junto a un grupo alquilo y } \mathrm{CH} 3 \text { en hidroaromaticos en el intervalo de } 22,5-24 \text { ppm, junto con anillos } \\
\text { naftalenicos (18-24 ppm). }\end{array}$ \\
\hline Pico numero 6 & $\begin{array}{l}\mathrm{CH} 2 \text { naftalenico, protegido por grupos } \alpha-\mathrm{CH} 2 \text { y grupos propilo, } \beta \text { - } \mathrm{CH} 3 \text { en grupos isopropilos, en el } \\
\text { intervalo de } 24-27,5 \mathrm{ppm} \text {. }\end{array}$ \\
\hline Pico numero 7 & $\begin{array}{l}\mathrm{CH} 2 \text { en grupos alquilos, } \mathrm{CH} 2 \text { unidos a anillos del grupo etileno, } \alpha-\mathrm{CH} 2 \text { y } \mathrm{CH}, \beta-\mathrm{CH} \text { en anillos } \\
\text { hidroaromaticos; naftalenicos } \mathrm{CH} 2 \text {, en el intervalo de } 27,5-37 \mathrm{ppm} \text {. }\end{array}$ \\
\hline Pico numero 8 & $\begin{array}{l}\text { Anillos unidos de metileno en el intervalo } 32-43 \text { ppm; } \mathrm{CH} \text { en grupos alquilos y anillos naftalenicos; } \mathrm{CH} 2 \\
\text { en grupos alquilos adyacentes a } \mathrm{CH} \text {, en el intervalo de } 37-60 \mathrm{ppm} \text {. }\end{array}$ \\
\hline Pico numero 9 & Aromáticos C-H, en el intervalo de 100-129.5 ppm con aromáticos ortho C-H a C-OH entre 100-115 ppm. \\
\hline Pico numero 10 & Aromáticos de enlace simple C-C y aromáticos C-NH, en el intervalo de 129 -148 ppm. \\
\hline Pico numero 11 & Aromáticos C-O, en el intervalo 148-168 ppm. \\
\hline
\end{tabular}

Los grupos funcionales con enlace aromático tienden a aumentar su intensidad, con el aumento del grado de maduración termal, debido al incremento de la condensación del kerógeno (FIGURA 9), que forman anillos aromáticos a partir de la perdida de oxigeno de los grupos funcionales que acompañan al carbono alifático; tal como se observa en los resultados del factor de aromaticidad (fa) calculado (TABLA 1).

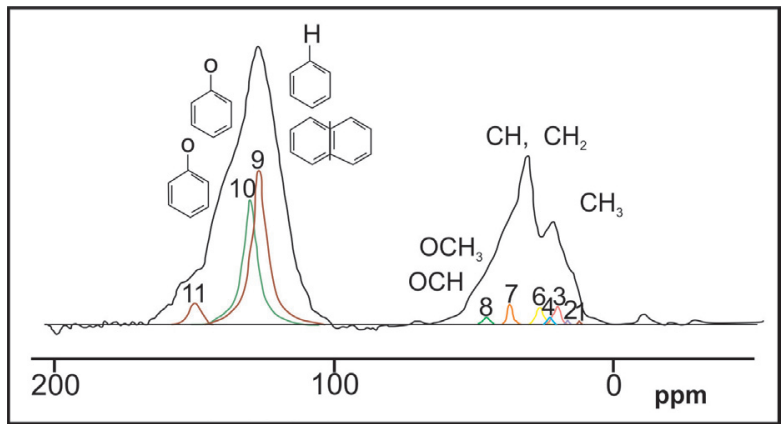

FIGURA 9. Deconvolución del espectro de RMN para la muestra del manto de carbón 1 (manto inferior), mediante el programa Dmfit de la Universidad D'orleans de Francia (versión gratuita).

\section{DISCUSIÓN DE RESULTADOS}

Los parámetros de madurez termal obtenidos mediante las técnicas de Ro, RMN (factor de aromaticidad), análisis proximal (materia volátil) y Tmax (FIGURAS 10, 11 y 12) muestran un factor de correlación aceptable entre los diferentes parámetros de madurez termal, lo cual permite realizar calibraciones a dichos parámetros. Este tipo de correlaciones son aplicables a nivel local, es decir, formaciones específicas y cuencas sedimentarias. En el presente caso se discute la correlación de maduración termal entre diferentes parámetros obtenidos a partir de carbones de la Formación Guaduas en la Cordillera Oriental.

Los parámetros de madurez termal fueron calibrados con respecto al Ro, que en el caso de los carbones presenta condiciones petrográficas óptimas para llevar a cabo las mediciones del maceral vitrinita. De esta manera se obtuvo una correlación aceptable entre la Ro y los parámetros de análisis proximal (materia volátil), Tmax y RMN (factor de aromaticidad), usando el parámetro Ro como una variable dependiente de los demás parámetros, para hallar un Ro calculado con base en las ecuaciones 2, 3 y 4 .

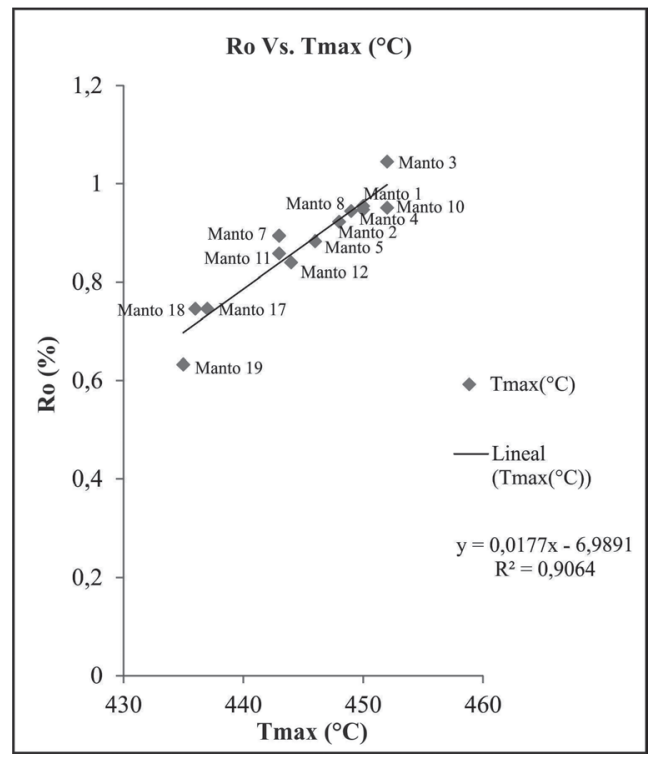

FIGURA 10. Relación de Reflectancia de vitrinita (\%Ro) vs La temperatura máxima de pirolisis Rock Eval (Tmax). 


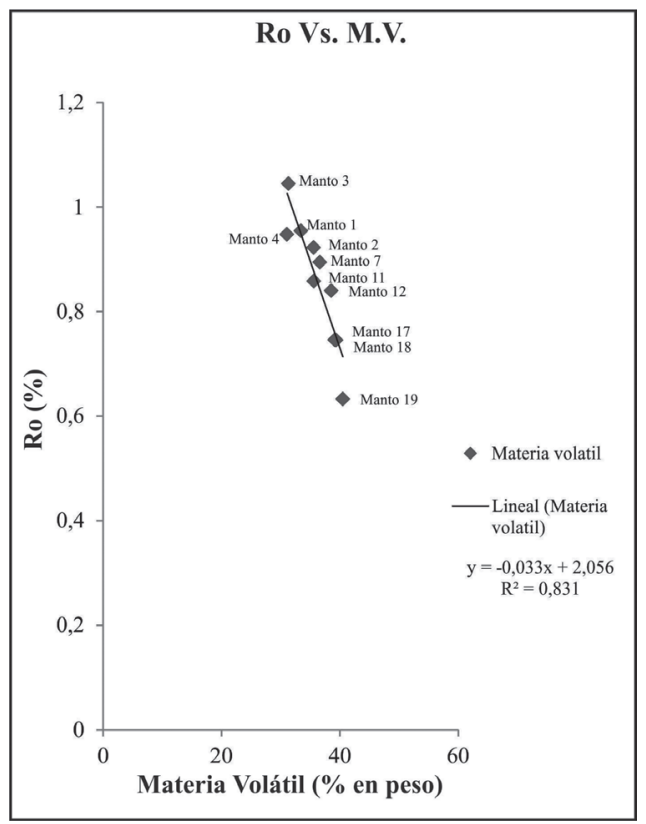

FIGURA 11. Relación de Reflectancia de vitrinita (\%Ro) Vs. Materia volátil (\%).

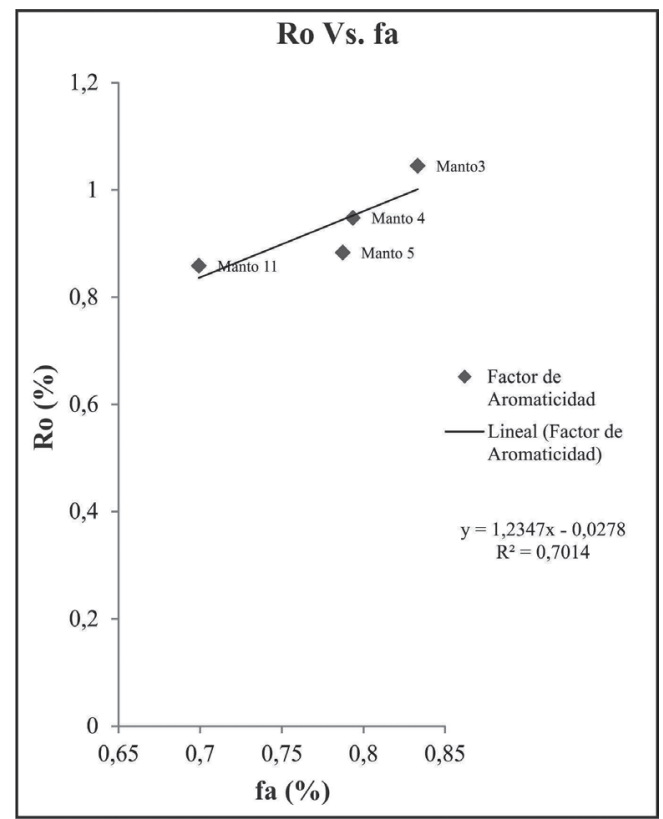

FIGURA 12. Relación de reflectancia de vitrinita (\%Ro) vs. el factor de aromaticidad (fa) calculado en RMN.

Las ecuaciones lineales obtenidas a partir de las FIGURAS 10, 11 y 12 de los parámetros mencionados son las siguientes:

Ro Calculado $=0,015($ Tmax $)-6,191$

Ro Calculado $=-0,033$ (M.V) $+2,056$

Ecuación (2)

Ro Calculado $=1,234(\mathrm{RMN})-0,027$
Donde: Ro, Reflectancia de vitrinita; M.V, materia volátil; RMN, equivale a los valores del factor de aromaticidad y Tmax, equivale a la Temperatura máxima medida durante la pirolisis Rock Eval.

Con base en las ecuaciones obtenidas, es posible calcular los valores de Ro para carbones de diversas áreas de la Cordillera Oriental, a partir de los datos de análisis proximales (materia volátil), Tmax y Rmn.

Para demostrar la aplicabilidad de las ecuaciones 2, 3 y 4 se utilizaron algunas áreas en las cuales afloran niveles de carbón pertenecientes a la Formación Guaduas, tales como: el sector de Tabio - río Frío, localizado dentro de la jurisdicción de los municipios de Cogua, Tausa, Zipaquirá y Pacho. En este sector afloran cinco niveles de carbón pertenecientes a la Formación Guaduas (Luna et al., 2004). Para los carbones de esta área se reporta una materia volátil 18,01\% (Luna et al., 2004), empleando la ecuación 3; se observa que el Ro calculado es igual a $1,4616 \%$, el cual coincide con los Ro medidos (entre 1,04 y $2,48 \%$ Ro). De igual forma, hacia el sector de Tabio - río Frío - Carmen de Carupa, se reportan datos de materia volátil de 20,80\% (Luna et al., 2004); aplicando la ecuación 3, se observa la misma tendencia en la cual el Ro calculado1,3696\% se ajusta dentro rango de Ro medido, el cual varía entre 1,04 y 2,48\% (Luna et al.,2004).

Otros ejemplos se pueden observar claramente en el sector de Cogua - Sutatausa - Guachetá, correspondiente al flanco occidental del Sinclinal Checua-Lenguazaque, en cual las secuencias de los mantos de carbón se localizan en la Formación Guaduas. Para este sector se reportan carbones con materia volátil 26,80\% (Luna et al., 2004); al emplear la ecuación 3 utilizando el porcentaje de materia volátil; se logra observar que el Ro calculado (1,1716\%), coincide con los rangos de Ro medidos en esta zona (entre $0,55 y$ y $2,71 \%$ Ro).

En el sector del páramo de La Bolda - Machetá, localizado entre los municipios de Machetá, Sueva, La Calera y Choachí, los mantos de carbón pertenecen a la Formación Guaduas y se encuentran principalmente en los sinclinales de Sueva, Machetá, La Bolsa y Sisga. En este sector los valores de reflectancia de vitrinita varían entre 0,41 a $0,80 \%$ (Luna et al., 2004); a su vez, estos mantos de carbón presentan un contenido de materia volátil de 35,59\% (Luna et al., 2004). Con base en estos datos se empleó la ecuación 3, obteniendo un Ro calculado de $0,88 \%$; este resultado coincide con el rango de variación del Ro medido para este sector. 


\section{CONCLUSIONES}

Los mantos de carbón de la Formación Guaduas en la sección del Triunfo, presentan una maduración termal que incrementa hacia la base de la formación, producto del aumento de la temperatura en profundidad, lo cual genera un incremento en la reacciones de aromatización del kerógeno; tal como lo indican los resultados de Ro, RMN, análisis proximal (materia volátil) y Tmax.

Al aumentar el grado de maduración termal de los mantos de carbón, los grupos funcionales y radicales que acompañan los enlaces de carbonos alifáticos del kerógeno disminuyen notablemente con el aumento de las reacciones de aromaticidad.

Los enlaces de carbonos alifáticos pierden principalmente el grupo funcional $\mathrm{CH} 2$ junto a un grupo alquilo, $\mathrm{CH} 3$ en hidroaromáticos y anillos naftalenicos, ubicado en el intervalo de desplazamiento químico de 22,5-24 ppm; con el aumento de la madurez termal de los mantos de carbón. Así mismo, se observó una paulatina disminución en los siguientes radicales y grupos funcionales que acompañan a dicho enlace con el incremento del grado de madurez termal:

- CH3 ó $\alpha$ - CH3 alejados de anillos aromáticos por dos anillos o grupos adyacentes, ubicados en el intervalo de desplazamiento químico de 11-15 ppm.

- $\quad \beta$ - $\mathrm{CH} 3$ en grupos etil, ubicado en el intervalo de desplazamiento químico de 15 a 18 ppm.

- $\alpha$ - CH3 no protegido por ningún anillo o grupo, ubicado en el intervalo de desplazamiento químico de 20.5 a 22.5 ppm.

- Anillos unidos de metileno $\mathrm{CH}$ en grupos alquilos y anillos naftalenicos; $\mathrm{CH} 2$ en grupos alquilos adyacentes a $\mathrm{CH}$, ubicados en el intervalo de desplazamiento químico de 37 a 60 ppm.

De igual forma, se estableció que los enlaces de carbonos aromáticos se enriquecen en los siguientes grupos funcionales: Aromáticos ortho $\mathrm{C}-\mathrm{H}$ a $\mathrm{C}-\mathrm{OH}$, ubicados en el intervalo de 100-129,5 ppm del espectro de RMN; Aromáticos de enlace simple C-C y aromáticos C-NH, ubicados en el intervalo de 129 a 148 ppm del espectro de RMN y Aromáticos C-O, ubicados en el intervalo de desplazamiento químico de 148 a 168 ppm ; con el aumento de la madurez termal de los mantos, aumentando las reacciones de aromatización del kerógeno.

El aumento de la madurez termal en los mantos de carbón de la sección del Triunfo muestra un aumento en el proceso de aromatización y deshidrogenación del kerógeno en los mantos inferiores de la secuencia (FIGURA 2 y 6), de acuerdo con el IH obtenido para estos. Así mismo, la elevada cantidad de hidrogeno disponible en el kerógeno de los mantos superiores, de acuerdo con el $\mathrm{IH}$, evidencia un proceso de aromatización y deshidrogenación más bajo comparado con los mantos inferiores.

La cantidad de materia volátil (\% en peso) presente en los diferentes mantos de carbón muestra la misma tendencia observada en el $\mathrm{IH}$, indicando un incremento en la perdida de materia volátil a medida que aumenta el grado de madurez termal, y por consiguiente el proceso de aromatización y deshidrogenación del kerógeno. Es por esta razón que los mantos inferiores presentan porcentajes de materia volátil más bajos comparados con los mantos superiores (FIGURA 6).

La metodología empleada en esta investigación puede ser aplicada en la identificación y evaluación de áreas prospectivas de yacimientos no convencionales. La correlación entre los parámetros de madurez termal usados en esta investigación (materia volátil, Tmax, RMN) permiten llevar a cabo calibraciones en los estados de maduración termal para formaciones especificas, usando el parámetro Ro como una variable dependiente de los demás parámetros, para hallar un Ro calculado.

Para la determinación del grado de madurez termal de la roca madre es indispensable usar diferentes técnicas de análisis geoquímicos, ya que la aplicación de una sola técnica analítica por sí sola no demuestra una alta confiabilidad. Es por tal razón, que esta investigación explica y demuestra la importancia de calibrar debidamente cada una de las técnicas utilizadas para la determinación de la madurez termal de la roca madre, a fin de que su aplicabilidad permita obtener datos veraces y confiables. De esta forma, se podría sustituir los valores de Ro y Tmax de Tissot and Welte (1984), los cuales se utilizan en forma universal sin tener en cuenta que estos parámetros deben ser calibrados para las diferentes formaciones y cuencas sedimentarias donde serán aplicados.

\section{AGRADECIMIENTOS}

Los autores expresan sus agradecimientos al Grupo de Investigación en Geología de Hidrocarburos y Carbones (G.I.G.H.C) por la financiación brindada para el desarrollo de esta investigación y por permitir el uso de los equipos de su laboratorio. A Humberto Carvajal Ortiz $(\mathrm{PhD})$ por sus sugerencias y revisiones, las cuales permitieron mejorar en gran medida el manuscrito. 


\section{REFERENCIAS}

Behar ,F., Vandenbroucke, M., Tang, Y., Marquis, F. and Espitalie, J. 1997. Thermal cracking of kerogen in open and closed systems: determination of kinetic parameters and stoichiometric coefficients for oil and gas generation. Organic Geochemistry, 26 (5/6): 321-339.

Bustin, R., and Guio, Y. 1999. Abrupt change (jumps) in reflectance values and chemical compositions of artificial charcoals and inertinite in coal, International Journal of Coal Geology, 38: 237-260.

Espitalié, J., et Marquis, F. 1992. La pyrolyse rock-eval et ses applications, deuxiéme partie. Revue de l'institute du Petrole, 40: 755-784.

García-González, M., MacGowan, D., and Surdam, R. 1993. Coal as a source rock of petroleum and gas - a comparison between natural and artificial maduration of the Almond Formation Coals, Greater Green River Basin in Wyoming. The Future of Energy Gases, U.S. Geological Survey Professional Paper, 1570: 405-436.

Gómez, J., Nivia, A., Montes, N., Jiménez, D., Tejada, M., Sepúlveda, M., Osorio, J., Gaona, T., Diederix, H., Uribe, H., y Mora, M. 2007. Atlas Geológico de Colombia, escala 1:500.000. INGEOMINAS.

Luna, L., Rodríguez, E., Sánchez, C., Renzoni, G., Hernández, E., Maldonado, J., Alba, C., Sanabria, A., y L-T- Geoperforaciones y Mneria Ltda. 2004. El Carbón Colombiano, Recursos, Reservas y Calidad. INGEOMINAS, 470p.

Miknis, F., Sullivan, M., Bartuska, V., and Maciel, G.1981. Cross-polarization magic angle spinning 13C NMR spectra of coals of varying rank. Organic Geochemistry, 3: 19-28.

Rincón, L., y Rocha, A. 2007. Estratigrafía de secuencias de la Formación Guaduas en la cuenca de Bogotá, Colombia. Tesis de grado, Universidad Industrial de Santander, Bucaramanga, Santander, Colombia.

Stéphane, G., Patrice, R., Jean-Jacques, D., Jean, C. and Patrice, V. 1981. Quantitative Carbon-13 and proton nuclear magnetic resonance spectroscopy of crude oil and petroleum products. I. Some rules for obtaining a set of reliable structural parameters. FUEL, 60 (3): 221-225.

Tissot, B., and Welte, D. 1984. Petroleum formation and occurrence, Second edition, Springer - Verlag, 699p.
Valenzuela, A., y García, M. 2002. El potencial de generación de gas y petróleo de los carbones de la Formación Guaduas, Tesis de grado. Universidad Industrial de Santander, Colombia. Biblioteca inventario $\mathrm{N}^{\circ} 104546$.

Vanderbroucke, M., and Largeau, C. 2007. Kerogen origin, evolution and structure, Organic Geochemistry, 38: 719-833.

Van Krevelen, D. 1993. Coal, Typology-PhysicsChemistry-Constitution, 3rd edition, Elsevier science publisher, 979p.

Werner, U., Lis, G., Mastalerz, M., and Schimmelmann, Arndt. 2005. Thermal maturity of type II kerogen from the New Albany Shale assessed by 13C CP/MAS NMR. Solid State Nuclear Magnetic Resonance, 27 (1-2): 140-148.

Trabajo recibido: mayo 26 de 2014

Trabajo aceptado: diciembre 11 de 2015

Manuscrito publicado en internet: diciembre 16 de 2015 